\title{
A Plastic Contamination Image Dataset for Deep Learning Model Development and Training
}

\author{
Mathew G. Pelletier * $\left(\mathbb{C}\right.$, Greg A. Holt ${ }^{\circledR}$ and John D. Wanjura \\ Agricultural Research Services, United States Department of Agriculture, Lubbock, TX 79404, USA; \\ Greg.Holt@usda.gov (G.A.H.); John.Wanjura@usda.gov (J.D.W.) \\ * Correspondence: Mathew.Pelletier@usda.gov
}

Received: 17 April 2020; Accepted: 19 May 2020; Published: 22 May 2020

\begin{abstract}
The removal of plastic contamination in cotton lint is an issue of top priority for the U.S. cotton industry. One of the main sources of plastic contamination appearing in marketable cotton bales is plastic used to wrap cotton modules on cotton harvesters. To help mitigate plastic contamination at the gin, automatic inspection systems are needed to detect and control removal systems. Due to significant cost constraints in the U.S. cotton ginning industry, the use of low-cost color cameras for detection of plastic contamination has been successfully adopted. However, some plastics of similar color to background are difficult to detect when utilizing traditional machine learning algorithms. Hence, current detection/removal system designs are not able to remove all plastics and there is still a need for better detection methods. Recent advances in deep learning convolutional neural networks (CNNs) show promise for enabling the use of low-cost color cameras for detection of objects of interest when placed against a background of similar color. They do this by mimicking the human visual detection system, focusing on differences in texture rather than color as the primary detection paradigm. The key to leveraging the CNNs is the development of extensive image datasets required for training. One of the impediments to this methodology is the need for large image datasets where each image must be annotated with bounding boxes that surround each object of interest. As this requirement is labor-intensive, there is significant value in these image datasets. This report details the included image dataset as well as the system design used to collect the images. For acquisition of the image dataset, a prototype detection system was developed and deployed into a commercial cotton gin where images were collected for the duration of the 2018-2019 ginning season. A discussion of the observational impact that the system had on reduction of plastic contamination at the commercial gin, utilizing traditional color-based machine learning algorithms, is also included.
\end{abstract}

Keywords: machine vision; plastic contamination; cotton; automated inspection

\section{Introduction}

The removal of plastic contamination in cotton lint is an issue of top priority for the U.S. cotton industry. One of the main sources of plastic contamination appearing in marketable cotton bales at the U.S. Department of Agriculture's classing office is from the plastic used to wrap cotton modules produced by the new John Deere round module harvesters. Despite diligent efforts by cotton ginning personnel to remove all plastic encountered during unwrapping of the seed cotton modules, plastic still finds a way into the cotton gin's processing system. Plastic contamination in cotton is thought to be a major contributor to the loss of a US $\$ 0.02 / \mathrm{kg}$ premium that U.S. cotton used to obtain on the international market due to its reputation as one of the cleanest cottons in the world. Current data now show that U.S. cotton is trading at a US $\$ 0.01 / \mathrm{kg}$ discount relative to the market, with a total loss of US $\$ 0.034 / \mathrm{kg}$ with respect to market conditions prior to the wide-spread adoption of plastic-wrapped 
cotton modules. The cost of this loss to U.S. producers is in excess of US\$750 million annually [1]. In order to help address this loss and mitigate plastic contamination at the cotton gin, inspection systems are being developed that utilize low-cost color cameras to observe plastic at various locations throughout the cotton gin for plastic wrap detection and control of plastic removal machinery.

This report covers the development of an image dataset that can be used to further improve modern detection systems that are needed to control removal machinery. The image dataset is included, as a supplemental, along with this report as an open-source resource to help further advance the science and lower the barriers of development. The annotated image dataset, provided with this technical note, is supplied in the Pascal VOC format and is ready for import into Tensorflow [2] or Keras [3] deep learning model training and development environments. The deep learning format was selected given the recent wide scope of rapid advancements demonstrated in a diverse range of disciplines [4-6]. The objective of this paper was to provide the background and relevant information in support of the auxiliary image dataset that is included with this paper. The value of a deep learning model is in no small measure associated with the image datasets utilized to build the models, as data collection requires an extensive amount of time and research funding. To ensure the value of an image dataset, the background information is critically important in terms of how the images are collected and under what specific conditions (lighting levels, lighting color-temperature, image sensor used, sensor settings etc.). This paper provides that key information so that future researchers looking at developing plastic contamination models can leverage this image dataset to achieve their objectives without having to duplicate the research that was undertaken to obtain the images presented herein.

\section{Materials and Methods}

To capture images, a prototype system was developed and deployed for a commercial cotton gin. To keep the optics clean in the high-dust environment of a cotton gin, a custom camera housing was developed that included an integral air knife directed across the optics to keep it clear of dust and debris (Figure 1).

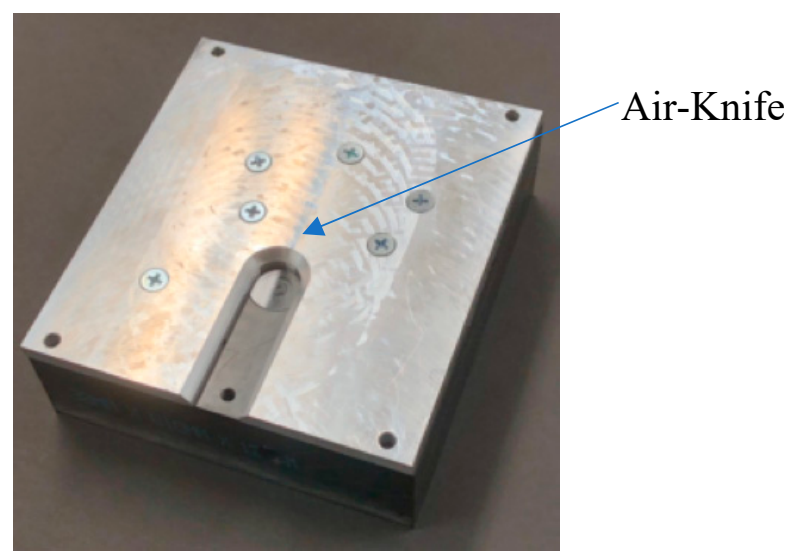

Figure 1. Camera housing design with cooling and integral air knife for keeping optics clean in high-dust environments.

The camera housing also held the computer node that was comprised of a low-cost embedded processing board with a Broadcom BCM2837 ARM central-processing-unit (CPU) that was configured to run Debian operating system (OS). The image acquisition software was written in $\mathrm{C}++$. The camera comprised a low-cost cell-phone rolling-shutter image sensor (Sony IMX219PQ). To minimize the pipeline data transport delay between the imager and the CPU, a fast camera serial interface (CSI) bus was selected to couple the camera to the mobile industry processor interface (MIPI) that is embedded in the CPU. With cotton flowing under the camera at $1 \mathrm{~m} / \mathrm{s}$, the imager was configured to run at 40 frames per second (FPS) with a resolution of $640 \times 480$ pixels and an exposure time of $500 \mathrm{~ms}$. 
To span the entire $2.4 \mathrm{~m}$ width of the cotton gin-stand feeder, a support frame was constructed to hold the lights and the camera housing. The lighting was configured to provide $5000 \mathrm{~K}$ illumination from 628 to 892 lux with a mean of 772 lux and a standard deviation of 110 lux (14\% of mean). The camera housings were spaced to provide six imaging nodes across the feeder, and the optics were configured to view $0.4 \mathrm{~m}$ of cotton along the long axis of the image in the horizontal axis. Figure 2 shows manual testing of the camera system to assess detectability during gin operation.

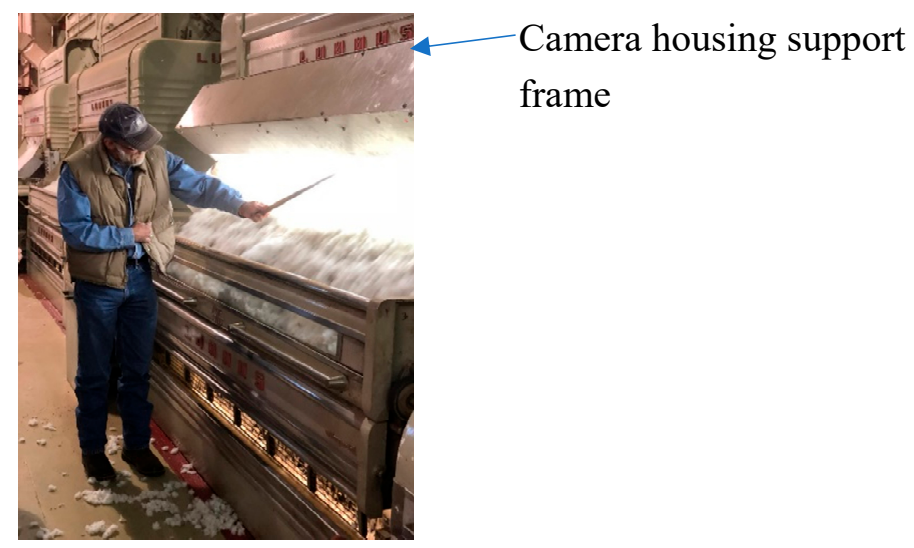

Figure 2. Field testing of the camera detection system in commercial cotton gin.

The camera detection system was run for the duration of the 2018 and 2019 cotton ginning seasons at a commercial cotton gin. Example images of plastic contamination captured by the system are shown in Figure 3. Leveraging these captured images, an image dataset was created and hand-edited to provide bounding boxes surrounding each item of interest in each and every image, such that the image dataset could be submitted to a deep learning model, via standard tools such as Keras, PyTorch, or Tensorflow. The image dataset annotations comprised three classes (plastic, cotton, and tray background) with:

- 401 occurrences of plastic contamination,

- 4751 occurrences of seed cotton

- 2847 occurrences of aluminum tray (background)

The seed cotton class included not only white seed cotton but also sticks, leaf trash, and cotton carpel segments (burs).

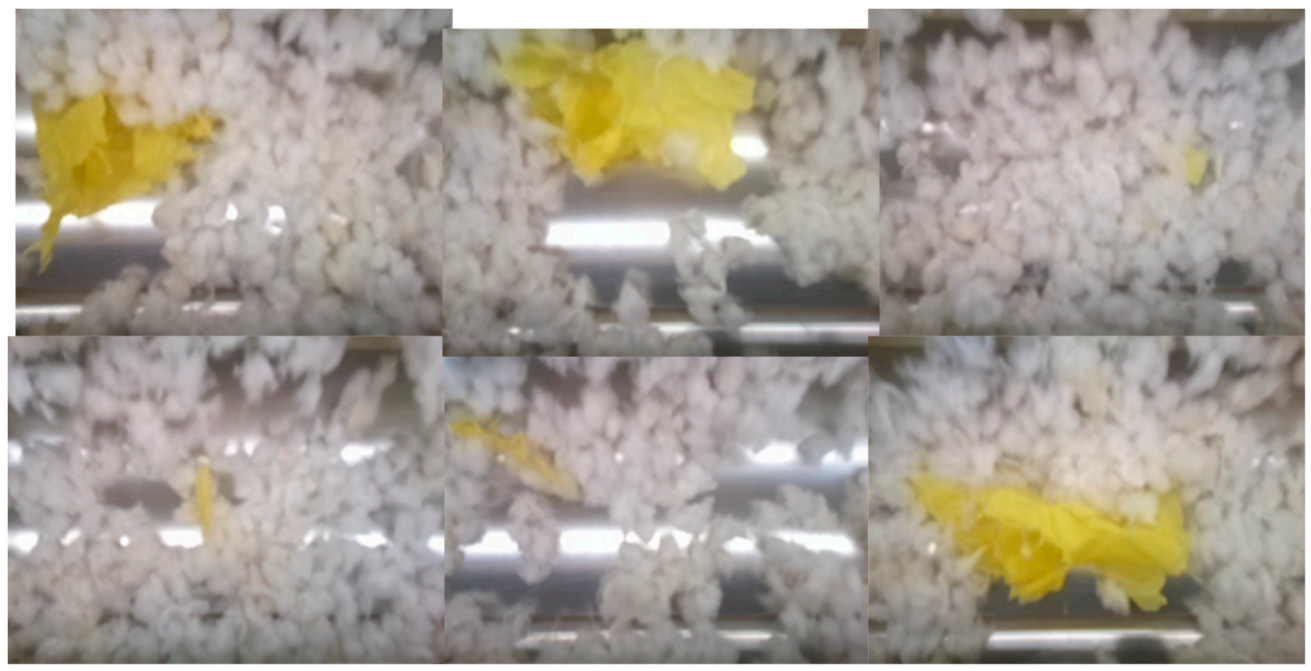

Figure 3. Plastic contamination images, captured by the prototype camera system, as deployed in a commercial cotton gin. 
While the current prototype detection system using traditional color classifiers is able to detect most of the normal module-wrap plastic contaminant colors, (blue, green, pink, and yellow), it struggles when cotton is more degraded and becomes yellow-spotted, as the overlap in colors is significant, leading to large numbers of false positives which is unacceptable. Classification rates have been found in commercial trials to vary from $90 \%$ in very clean white cotton to $75 \%$ in cotton with high levels of leaves, burs, sticks, and yellow-spotted cotton. Other significant contaminant colors neglected in current image classifiers are white grocery bags, as against the white cotton background there is no way to separate the two. Other problematic colors are white and black plastic, as in the $\mathrm{L}^{*} \mathrm{a}^{*} \mathrm{~b}^{*}$ color-space used by the author's classifier, both shows up in exactly the same place as cotton $(a=0$, $\mathrm{b}=0$ ). While use of the luminance channel is an option, in practice, the white cotton that is in shadows on edges of the cotton bolls are grey, which is also very near center $(a=0, b=0)$. Thus, while black is an easy color for the human visual system, a machine vision system utilizing a color space such as $\mathrm{L}^{*} \mathrm{a}^{*} \mathrm{~b}^{*}$ or similar color-spaces such as: hue-saturation-value, HSV; or hue-saturation-brightness, HSB; or hue-saturation-lightness, HSL, all will have similar difficulties. Hence, there is still a significant need for improvements in classifier development. A classifier based on deep learning that responds primarily to texture differences is hoped to have the potential to solve some of these issues and thereby improve classification rates.

To get to the next level of detectability, white or near-white plastic against a background of white cotton, the detection method needs to transition from traditional machine learning algorithms that utilize color difference-based detection to one that relies on texture differences. For deep learning training of deep-learning models such as convoluational-neural-networks, CNNs, a large dataset is necessary where objects from each class of interest must be identified and marked out as a sub-image portion in a bounding-box, where the coordinates of this sub-image bounding-box are provided to the $\mathrm{CNN}$ training algorithms through an annotation text file that provides bounding-box coordinates along with an associated class for each object in every image. Figure 4 shows an example of one of the annotated images where the different colors for each bounding box represent a different class (yellow: tray, red: cotton, purple: plastic). As texture differences represent the proposed metric of detection, for the deep learning algorithms the included dataset with yellow plastic can be used for exploring this by converting the color images to gray-scale during the importation submission to the deep learning tools. That will ensure the deep learning algorithms simply do not utilize color in the back-propagation training of the CNN's classifiers.

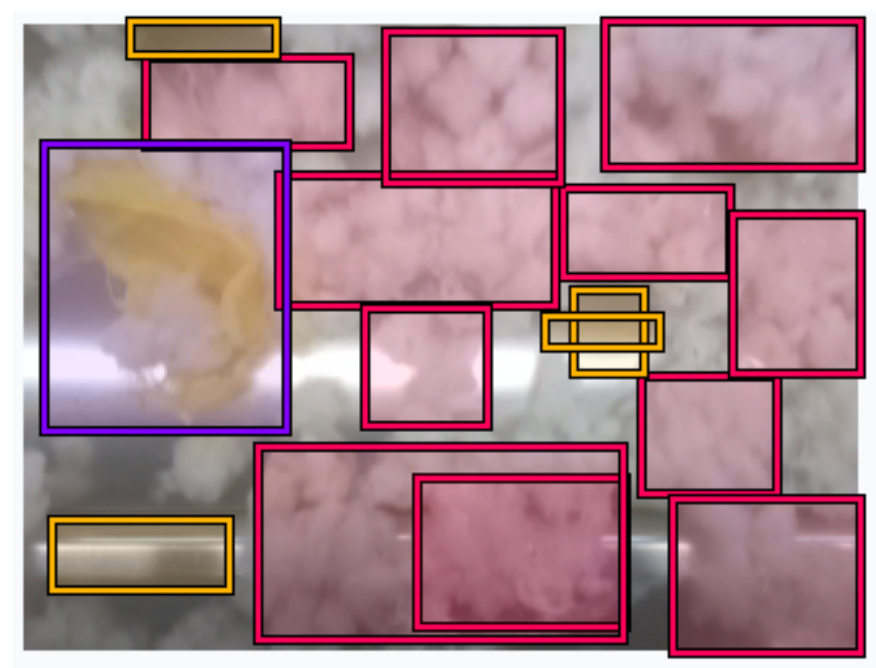

Figure 4. Deep learning annotated image, with each colored bounding-box representing a different class object. This annotated image is one in the large image dataset that is included as an attachment to this technical note. 


\section{Utilization}

As capturing real-world plastic generated in a cotton gin is an expensive and time-consuming task, it is hoped that this image dataset will provide a training set for any team wanting to pursue the development of detection systems targeted for use in cotton gins (either traditional color based or CNNs). The images were all collected during the normal course-of-business of ginning cotton in a commercial cotton gin. These images are true representatives of what a system would be expected to be able to classify using similar low-cost hardware and software. The software will be released under an open-source library in a subsequent publication covering the specifics of the software design.

\section{Conclusions}

This technical note covers the specifics on how an extensive image dataset for plastic contamination detection at a cotton gin was collected, and provides that image dataset in a deep learning ready format suitable for use in Keras or Tensorflow deep learning toolsets as well as exploration of new classifiers using traditional machine-learning algorithms. The image dataset is provided under an open-source license with the hope of stimulating competitive designs suitable for use in the cotton industry for detection and removal of plastic contamination.

Supplementary Materials: The annotated image dataset is available as an attachment to this manuscript. The following are available online at http://www.mdpi.com/2624-7402/2/2/21/s1.

Author Contributions: All authors contributed materially to the development of this article and the associated image dataset. All authors have read and agreed to the published version of the manuscript.

Funding: This research was funded by Cotton Incorporated grant number 18-239.

Conflicts of Interest: The authors declare no conflict of interest.

Disclaimer: Mention of a product or tradename in this article does not constitute an endorsement by the USDA-ARS over other compatible products. Products or trade names are listed for reference only. USDA is an equal opportunity provider and employer.

\section{References}

1. Jon, D. Economic Impact of Plastic Contamination in Cotton; Cotton Incorporated Economcs. Written correspondence with authors; Cotton Incorporated: Cary, NC, USA, 2019.

2. Abadi, M.; Agarwal, A.; Barham, P.; Brevdo, E.; Chen, Z.; Citro, C.; Corrado, G.; Davis, A.; Dean, J.; Devin, M.; et al. TensorFlow: Large-Scale Machine Learning on Heterogeneous Systems. 2015. Available online: http://tensorflow.org (accessed on 4 March 2020).

3. Chollet, F. Keras. Available online: https://keras.io (accessed on 4 March 2020).

4. Bakator, M.; Radosav, D. Deep Learning and Medical Diagnosis: A Review of Literature. Multimodal Technol. Interact. 2018, 2, 47. [CrossRef]

5. Griffiths, D.; Boehm, J. A Review on Deep Learning Techniques for 3D Sensed Data Classification. Remote Sens. 2019, 11, 1499. [CrossRef]

6. Alom, M.; Taha, T.; Yakopcic, C.; Westberg, S.; Sidike, P.; Nasrin, M.; Hasan, M.; Van Essen, B.C.; Awwal, A.A.S.; Asari, V.K. A State-of-the-Art Survey on Deep Learning Theory and Architecutures. Electronics 2019, 8, 292. [CrossRef]

(C) 2020 by the authors. Licensee MDPI, Basel, Switzerland. This article is an open access article distributed under the terms and conditions of the Creative Commons Attribution (CC BY) license (http://creativecommons.org/licenses/by/4.0/). 